\title{
Which Improvised Tourniquet Windlasses Work Well and Which Ones Won't?
}

\author{
John F. Kragh Jr, MD; Timothy E. Wallum, BS; James K. Aden III, PhD; Michael A. Dubick, PhD; \\ David G. Baer, PhD \\ From the Damage Control Resuscitation, US Army Institute of Surgical Research, JBSA Fort Sam Houston, TX (Drs Kragh and Dubick); the Burn \\ Center, US Army Institute of Surgical Research, JBSA Fort Sam Houston, TX (Mr Wallum); the Statistics Department, US Army Institute of \\ Surgical Research, JBSA Fort Sam Houston, TX (Dr Aden); and the Research Directorate, US Army Institute of Surgical Research, JBSA Fort Sam \\ Houston, TX (Dr Baer).
}

Objective.-Improvised tourniquets in first aid are recommended when no scientifically designed tourniquet is available. Windlasses for mechanical advantage can be a stick or pencil and can be used singly or multiply in tightening a tourniquet band, but currently there is an absence of empiric knowledge of how well such windlasses work. The purpose of the present study was to determine the performance of improvised tourniquets in their use by the type and number of windlasses to improve tourniquet practice.

Methods. - A simulated Leg Tourniquet Trainer was used as a manikin thigh to test the effectiveness of improvised tourniquets of a band-and-windlass design. Two users made 20 tests each with 3 types of windlasses. Tests started with 1 representative of a given type (eg, 1 pencil), then continued with increasing numbers of each windlass type until the user reached $100 \%$ effectiveness as determined by cessation of simulated blood flow. Windlass types included chopsticks, pencils, and craft sticks.

Results.-Effectiveness percentages in stopping bleeding were associated inversely with breakage percentages. Pulse stoppage percentages were associated inversely with breakage. The windlass turn numbers, time to stop bleeding, the number of windlasses, and the under-tourniquet pressure were associated inversely with breakage. The windlass type was associated with breakage; at 2 windlasses, only chopsticks were without breakage. Of those windlass types that broke, $20.7 \%$ were chopsticks, $26.1 \%$ were pencils, and $53.2 \%$ were craft sticks.

Conclusions.-A pair of chopsticks as an improvised tourniquet windlass worked better than pencils or craft sticks.

Key words: first aid, resuscitation, damage control, hemorrhage, trauma, shock

\section{Introduction}

First aid tourniquets have become more commonly used, for example, in the aftermath of the Boston Marathon bombing in 2013. ${ }^{1}$ After more than a decade of war, with more veterans returning to the United States with tourniquet experience, and increasing media publicity of civilian use of tourniquets in the United States, the

Disclaimer: The opinions or assertions contained herein are the private views of the authors and are not to be construed as official or reflecting the views of the Department of Defense or United States government. The authors are employees of the US government. This work was prepared as part of their official duties and, as such, there is no copyright to be transferred.

Corresponding author: John F. Kragh Jr, MD, US Army Institute of Surgical Research, 3698 Chambers Pass, Suite B, JBSA Fort Sam Houston, TX 78234-7767 (e-mail: john.f.kragh.civ@mail.mil). potential benefits to the civilian sector are becoming recognized. To control hemorrhage from compressible extremity wounds, improvised tourniquets are recommended when no commercially designed tourniquet is available. $^{2}$ Preliminary evidence from war indicates that improvised tourniquets challenge the user to make them both safe and effective. ${ }^{2}$ The common type of improvised tourniquet is of a band-and-windlass design where a band is twisted and tightened by a rodlike windlass. The windlass gives mechanical advantage in tightening the band. Types of windlasses may include sticks and pencils, which can be used singly or multiply. However, evidence of efficacy of improvised tourniquets is based mostly on opinions of clinicians with experience in tourniquets. ${ }^{2}$ We were unable to find datasets from which the US Army's lesson plan for improvised 
Table 1. Effective tests by windlass type, windlass number, and user

\begin{tabular}{|c|c|c|c|c|c|}
\hline Windlass type & $\begin{array}{l}\text { Windlass } \\
\text { number }\end{array}$ & $\begin{array}{l}\text { Tests per } \\
\text { user }(n)\end{array}$ & $\begin{array}{l}\text { Effective tests, } \\
\text { user } 1(n)\end{array}$ & $\begin{array}{c}\text { Effective tests, } \\
\text { user } 2(n)\end{array}$ & $\begin{array}{c}\text { Effectiveness percentage } \\
(\text { total tests }, n)\end{array}$ \\
\hline \multirow[t]{2}{*}{ Chopstick } & 1 & 20 & 10 & 7 & $42 \%(40)$ \\
\hline & 2 & 20 & 20 & 20 & $100 \%(40)$ \\
\hline \multirow[t]{4}{*}{ Pencil } & 1 & 20 & 19 & 2 & $52 \%(40)$ \\
\hline & 2 & 20 & 19 & 18 & $92 \%(40)$ \\
\hline & 3 & 20 & 20 & 13 & $82 \%(40)$ \\
\hline & 4 & 20 & NA & 20 & $100 \%(20)$ \\
\hline \multirow[t]{4}{*}{ Craft stick } & 1 & 20 & 0 & 0 & $0 \%(40)$ \\
\hline & 2 & 20 & 12 & 12 & $60 \%(40)$ \\
\hline & 3 & 20 & 20 & 18 & $95 \%(40)$ \\
\hline & 4 & 20 & NA & 20 & $100 \%(20)$ \\
\hline
\end{tabular}

NA, not applicable.

tourniquet use was developed. Although the Army's lesson plan is detailed in technique steps, and many of the claims seem like common sense, we wished to empirically explore the issue of improvised tourniquet use to identify opportunities for improved care.

There is very little literature available for the layman outdoor enthusiast concerning use of improvised tourniquets in the field. ${ }^{3-8}$ Hence, the purpose of the present study is to provide new knowledge to both the novice and to professionals for use and performance of improvised tourniquets by testing various types and numbers of windlasses.

\section{Methods}

This study was conducted under a protocol reviewed and approved by the regulatory office of the US Army Institute of Surgical Research. Tourniquet users were a pair of investigators familiar with military tourniquet training and clinical use of tourniquets. Both investigators were experienced in tourniquet research and the use of the manikin.

The investigators used the HapMed Leg Tourniquet Trainer (CHI Systems, Fort Washington, PA, USA)-a right-thigh body segment (leg number 000F) with an amputation injury just proximal to the knee-in a manner similar to previous reports. ${ }^{9,10}$ The medial hippelvic area had a computer interface that included a smart-

phone-like touchpad software (version 1.9) internal to the manikin. This device allowed the leg to be used independently and to be operated by user input through finger touch on the pad.

The system reported blood loss volume as calculated using a linear equation from the arterial capacity and number of pulses before hemorrhage control. The touchpad readout of each iteration showed the results, which included effectiveness of bleeding control as evidenced by the above-noted light display, time to hemorrhage control, pressure exerted under the tourniquet, and volume of blood loss. Time to hemorrhage control started when the iteration began, and stopped when the manikin sensed that the thigh was losing no more blood or when the windlass broke. Complete effectiveness $(100 \%)$ was considered to be cessation of blood loss and the termination of a distal pulse of the affected extremity.

The tourniquet was an improvised tourniquet involving a band-and-windlass design in which the band was a triangular $(37 \times 37 \times 52$ inches $)$ cloth made of cotton (muslin; Elwyn Inc, Elwyn, PA, USA). This bandage is a common US military item used for multiple first aid purposes (NSN 6510-00-201-1755). After removing it from its package, the users would partially unfold the olive drab bandage until it was 3.5 inches wide and 52 inches long. Users tightened the tourniquet around the thigh until distal simulated bleeding was believed to have stopped, based on visual inspection of the lights and palpitation for the distal pulse in the device. One windlass turn was a $180^{\circ}$ arc, which is the limit of wrist supination in turning the windlass.

The present study was an experiment to determine tourniquet performance as differentiated by type of windlass and the number of that type needed to be effective. We selected windlass types that varied in shape, size, and toughness so as to see potentially varied performance. Windlass types included bamboo chopsticks, pencils, and craft sticks (also known as Popsicle sticks). Tourniquet users tested performance in sets of 20 iterations. Each set of 20 iterations of testing was per user with each type of windlass. Users started with 1 windlass for each type. To determine when 
Table 2. Results of windlass breakage by effectiveness in stopping simulated blood flow

\begin{tabular}{lcccc}
\hline & Bleeding & \multicolumn{4}{c}{ Breakage } \\
\cline { 3 - 5 } Conditions & stopped & No & Yes & Total \\
\hline Iterations or attempts & No & 12 & 99 & 111 \\
$\quad$ Total attempts (\%) & & 3.3 & 27.5 & 30.8 \\
$\quad$ Ineffective attempts (\%) & & 10.8 & 89.2 & \\
Iterations or attempts & Yes & 237 & 12 & 249 \\
$\quad$ Total attempts (\%) & & 65.8 & 3.3 & 69.2 \\
Effective attempts (\%) & & 95.2 & 4.8 & \\
Total & & 249 & 111 & 360 \\
\hline
\end{tabular}

effectiveness would reach $100 \%$ in a set for each user for each windlass type, users proceeded with each successive set with an increasing number of windlasses until the user eventually reached $100 \%$ effectiveness for the set. Once the user reached $100 \%$ effectiveness for a type of windlass, the user switched to another windlass type and restarted the set progression. There were 2 tourniquet users.

Statistical analysis included use of descriptive statistics. We used contingency tables to compare variables (eg, breakage [yes/no] by hemorrhage control [yes/no]). A $\chi^{2}$ test was used to assess for differences. Analysis of variance was used to analyze the effects of tourniquet use on factors of interest. Analyses allowed for detection of intervariable associations, eg, if there was an association between the windlass type and hemorrhage control. Logistic regression was used to model the occurrence of breakage (yes/no).

\section{Results}

The only windlass type to be effective for all tests with the least number of windlasses was chopsticks, with 2 chopsticks being $100 \%$ effective for both users (Table 1). One user was able to make 3 windlasses effective $100 \%$ for both pencils and craft sticks, whereas the other user

Table 3. Results of windlass breakage by windlass turn number

\begin{tabular}{lrcr}
\hline & \multicolumn{3}{c}{ Breakage } \\
\cline { 2 - 4 } Turn number & No & Yes & Total \\
\hline 1 & 40 & 61 & 101 \\
2 & 143 & 34 & 177 \\
3 & 52 & 13 & 65 \\
4 & 14 & 3 & 17 \\
Total & 249 & 111 & 360 \\
\hline
\end{tabular}

Data represent sum of all iterations, users, and windlasses.
Table 4. Results of windlass breakage by windlass type

\begin{tabular}{lrcr}
\hline & \multicolumn{3}{c}{ Breakage } \\
\cline { 2 - 4 } Windlass type & No & Yes & Total \\
\hline \multirow{2}{*}{ Chopstick } & 57 & 23 & 80 \\
Pencil & 111 & 29 & 140 \\
Craft stick & 81 & 59 & 140 \\
Total & 249 & 111 & 360 \\
\end{tabular}

Data represent sum of all iterations, users, and windlasses.

needed 4 windlasses for both pencils and craft sticks to achieve $100 \%$ effectiveness.

When all 3 types of windlasses are considered, effectiveness (absence of pulse and blood flow) was associated inversely with breakage $(P<.0001$; Table 2$)$. With but rare exception (3.3\%), if the windlass broke, it was ineffective, and if it remained intact, then pulse and blood flow ceased (Table 1). The windlass turn numbers were associated inversely with breakage percentages $(P$ $<.0001$; Table 3). However, the results were complex as windlass type and windlass count were confounded. For example in 1 windlass type, craft sticks, low windlass turn numbers were associated with high breakage percentages, and this low-high association was true with only 1 craft stick as a windlass. Otherwise there was an association of low windlass turn numbers with low breakage percentages.

The breakage percentages of the 2 tourniquet users were not statistically different from each other $(P=$ .7). The breakage percentages by turn direction were not statistically different from each other $(P=.7)$, but each of the 2 users turned the windlass in different directions uniformly, so user identity and turn direction in effect became indistinguishable in the statistical model.

Windlass type was associated with percent breakage $(P<.0005 ;$ Table 4). Of those windlass types that broke, $20.7 \%$ were chopsticks, $26.1 \%$ were pencils, and $53.2 \%$ were craft sticks.

Table 5. Results of windlass breakage by windlass number

\begin{tabular}{lrcr}
\hline & \multicolumn{3}{c}{ Breakage } \\
\cline { 2 - 4 } Windlass number & No & Yes & Total \\
\hline & 38 & 82 & 120 \\
2 & 102 & 18 & 120 \\
3 & 69 & 11 & 80 \\
4 & 40 & 0 & 4 \\
Total & 249 & 111 & 360 \\
\hline
\end{tabular}

Data represent sum of all iterations, users, and windlasses. 
When the windlass number was pooled for all types of windlass, the windlass number was associated inversely with breakage percentages $(P<.0001$; Table 5$)$. Higher windlass numbers broke less often than lower numbers. The time to stop bleeding for iterations with breakage was longer than the time to stop bleeding for iterations without breakage $(P<.0001)$. The pressure developed under the tourniquet for iterations with breakage was less than the pressure for iterations without breakage $(P<$ .0001). For time to cessation of bleeding, windlass number, user identity, and the interaction of windlass type with windlass number were predictors of this time $(P<.0004)$. For pressure at cessation of bleeding, windlass type, windlass number, user identity, and the interaction of windlass type with windlass number were predictors of this pressure $(P<.0007)$. For loss of blood volume, windlass type, windlass number, user identity, and the interaction of windlass type with windlass number served as predictors $(P<.0015)$. Finally, for breakage itself using a multivariate logistic regression, windlass type, windlass number, and user identity were all predictors $(P<.0245)$.

\section{Discussion}

The major finding of the present study was that these 3 windlass types performed differently; chopsticks performed better than pencils or craft sticks. Two chopsticks reached $100 \%$ effectiveness: chopsticks were the only type to succeed completely at that number of windlasses. The results imply that the mechanical properties of these windlasses differed.

The first minor finding was that the number of windlasses affected performance: if the number was sufficient to avoid breaking, then effectiveness rates were reliably high. If windlasses broke, then effectiveness rates were uniformly dismal. The breakage points were routinely at the point of maximal shear at the windlass-band interface. If hemorrhage had been controlled at the time of breakage, then the time of breakage coincided with the time of loss of hemorrhage control. The proportions of effective iterations by windlass number showed a coherent and expected stepwise rise with each added windlass number.

The second minor finding from our data was that arterial occlusion by improvised tourniquet use was not reliably attained because ineffectiveness percentages were high. This differs from previous experience, which was more favorable after the Boston explosions, ${ }^{1}$ but windlasses were not apparently used out-of-hospital in Boston and their tourniquets may have been venous. ${ }^{2}$ Tourniquets that close off venous flow are venous tourniquets, and by definition they do not close off arteries and are not arterial tourniquets; venous tourniquets are ineffective in hemorrhage control.

The third minor finding was that turn number was inversely associated with breakage percentage. The association was mostly attributable to the fact that the windlass type and windlass count were confounded in the relationship among breakage, turn number, windlass type, and windlass count. For example, 39 of the 61 breakages after only 1 turn were from using only 1 craft stick.

New hypotheses for further research include screening for better windlasses or deselecting worse windlasses, band types may be screened or deselected, and intersubject variability may be explored further.

Limitations of the present report are related to the narrow scope of the experimental design. The manikin experiment did not look at clinical care complexity but focused on the controlled variables of interest.

\section{Conclusions}

In summary, the present study reports a manikin experiment that found that a pair of chopsticks as an improvised tourniquet windlass worked better than pencils or craft sticks.

\section{Acknowledgments}

This project was funded with internal USAISR and Defense Health Program funds. Dr. Harold Klemcke aided in manuscript preparation.

\section{References}

1. Caterson EJ, Carty MJ, Weaver MJ, Holt EF. Boston bombings: a surgical view of lessons learned from combat casualty care and the applicability to Boston's terrorist attack. J Craniofac Surg. 2013;24:1061-1067.

2. Kragh JF Jr, Walters TJ, Baer DG, et al. Practical use of emergency tourniquets to stop bleeding in major limb trauma. J Trauma. 2008;64(suppl):S38-S49.

3. Lakstein D, Blumenfeld A, Sokolov T, et al. Tourniquets for hemorrhage control on the battlefield: a 4-year accumulated experience. J Trauma. 2003;54(suppl):S221S225.

4. King DR, van der Wilden G, Kragh JF Jr, Blackbourne LH. Forward assessment of 79 prehospital battlefield tourniquets used in the current war. J Spec Oper Med. 2012;12:33-38.

5. Dayan L, Zinmann C, Stahl S, Norman D. Complications associated with prolonged tourniquet application on the battlefield. Mil Med. 2008;173:63-66.

6. Swan KG Jr, Wright DS, Barbagiovanni SS, Swan BC, Swan KG. Tourniquets revisited. J Trauma. 2009;66:672-675.

7. Rich NM. Vascular trauma historical notes. Perspect Vasc Surg Endovasc Ther. 2011;23:7-12. 
8. Guo JY, Liu Y, Ma YL, Pi HY, Wang JR. Evaluation of emergency tourniquets for prehospital use in China. Chin J Traumatol. 2011;14:151-155.

9. Clumpner BR, Polston RW, Kragh JF Jr, et al. Single versus double routing of the band in the Combat
Application Tourniquet. J Spec Oper Med. 2013;13: 34-41.

10. Polston RW, Clumpner BR, Kragh JF Jr, Jones JA, Dubick MA, Baer DG. No slackers in tourniquet use to stop bleeding. J Spec Oper Med. 2013;13:12-19. 